\title{
Cenário do mapeamento de áreas contaminadas nos Estados do Rio de Janeiro e São Paulo.
}

Fabiana Pereira Coelho'; Ubirajara Hutter Carvalho²; Thamires Henrique Teles da Silva ${ }^{3}$

Resumo: A necessidade de ocupação de áreas com indícios de contaminação foi determinante para o avanço dos estudos e criação de normas e regulamentos para o seu gerenciamento. No Brasil, o marco das investigações destes locais foi o Manual de Gerenciamento de Áreas Contaminadas, elaborado pela Companhia Ambiental de São Paulo (CETESB) com o apoio da cooperação técnica alemã (GIZ) e a publicação da RESOLUÇÃO CONAMA 420 em 2009. Nela, umas das principais etapas é a criação de um cadastro de áreas contaminadas que deve ser publicado pelos órgãos ambientais estaduais, a fim de mostrar para a sociedade sua localização e principais características. Sendo assim este trabalho possui o objetivo de analisar o cenário atual dos estados de São Paulo e Rio de Janeiro, em relação à classificação das áreas contaminadas segundo a CONAMA 420, de modo a possibilitar um comparativo entre os estados, com relação à evolução e atual situação das mesmas.

Abstract: Rules and regulations for contaminated sites management came into practice in Brazil due to the fact of the interest in their re-use. The Manual for Contaminated Sites Management, elaborated by the environmental agency of São Paulo (CETESB) with the support of the German technical cooperation (GIZ), was a milestone toward the management of contaminated sites in Brazil. This manual is the basis for creating the main national standard for contaminated site management, the resolution CONAMA 420, published in 2009. A data bank of contaminated sites is regulated by this CONAMA resolution and obligates the state's environmental agencies to publish the information regarding site location and characteristics for the benefit of and transparency towards civil society. This study analyzes the current scenario of contaminated sites in Rio de Janeiro (RJ) and São Paulo (SP) according to the CONAMA 420 classification. It compares and evaluates its evolution and assesses the current situation between the states from which data was collected.

Palavras-Chave: Áreas contaminadas, Cadastro de Áreas Contaminadas.

1 Instituto SENAI de Tecnologia Ambiental - Rua Moraes e Silva, 53 - Bloco 9 - Maracanã/RJ, Cep 20271-030.
(fpcoelho@firjan.org.br), 3978-6148
2 Instituto SENAI de Tecnologia Ambiental - Rua Moraes e Silva, 53 - Bloco 9 - Maracanã/RJ, Cep 20271-030.
(ucarvalho@firjan.org.br), $3978-6125$
3 Instituto SENAI de Tecnologia Ambiental - Rua Moraes e Silva, 53 - Bloco 9 - Maracanã/RJ, Cep 20271-030.
(Thhsilva@firjan.org.br), 39786175 


\section{INTRODUÇÃO}

Os constantes avanços nos estudos de áreas contaminadas, as legislações vigentes, ações dos órgãos públicos e as necessidades de expansão empresarial, principalmente motivado pela necessidade de reabilitar e recuperar áreas já degradas, com presença de passivos ambientais, demandaram a necessidade de conhecer qual é o cenário real dos Estados mais desenvolvidos, em relação as áreas contaminadas.

Segundo o INEA (2014) uma área com contaminação é definida pela presença de substância(s) química(s) no ar, água, solo ou sedimento, decorrentes de atividades antrópicas, em concentrações tais que restrinjam a utilização desse recurso ambiental para os usos atual ou pretendido, definidas com base em avaliação de risco à saúde humana, assim como aos bens a proteger, em cenário de exposição padronizado ou específico.

Essa necessidade de ocupação de áreas com indícios de contaminação foi determinante para o avanço dos estudos e criação de normas e regulamentos para o seu gerenciamento. No Brasil, o marco das investigações destes locais foi o Manual de Gerenciamento de Áreas Contaminadas, elaborado pela Companhia Ambiental de São Paulo (CETESB) com o apoio da cooperação técnica alemã. Este manual foi a base para criação da principal norma nacional para o gerenciamento de áreas contaminadas, a Resolução CONAMA 420, publicada em 2009. Nela, umas das principais etapas é a criação de um cadastro de áreas contaminadas que deve ser publicado pelos órgãos ambientais estaduais, a fim de mostrar para a sociedade sua localização e principais características. Os cadastros de São Paulo e Rio de Janeiro, únicos estados que os publicaram, foram as bases deste estudo.

Em atendimento a necessidade de regulamentação específica a nível federal, em 2009 foi promulgada a Resolução CONAMA 420, a qual dispõe sobre critérios e valores orientadores de qualidade do solo (valores de prevenção e valores de intervenção), quanto à presença de substâncias químicas e estabelece diretrizes para o gerenciamento ambiental de solos contaminados por essas substâncias em decorrência de atividades antrópicas. Sua publicação teve significativo avanço para uma maior atuação dos órgãos ambientais no controle e acompanhamento do gerenciamento de áreas contaminadas nos estados da nação.

Além da legislação federal, Estados como São Paulo e Rio de Janeiro, possuem legislações Estaduais específicas para áreas contaminadas, em São Paulo vigora a Lei n 13.577 , de 08 de julho de 2009, e no Rio de Janeiro a Resolução CONEMA n ${ }^{\circ} 44$ de 14 de dezembro de 2012. 


\section{OBJETIVO}

O presente estudo tem por objetivo analisar o cenário atual dos Estados de São Paulo e Rio de Janeiro em relação às atividades geradoras da contaminação, à classificação das áreas contaminadas, a evolução do número destas áreas contaminadas registradas de modo a possibilitar um comparativo entre os estados, com relação à evolução e atual situação das mesmas.

\section{METODOLOGIA}

Para a avaliação do panorama atual das áreas contaminadas nos Estados de São Paulo e Rio de Janeiro, foram consultados os cadastros publicados de áreas contaminadas dos estados de São Paulo e Rio de Janeiro. O inventário de áreas contaminadas apresenta a classificação das mesmas conforme a Resolução CONAMA 420, a evolução do seu quantitativo, identificação e cadastramento de novas áreas. Em São Paulo, o cadastro é elaborado pela CETESB, tendo sido divulgado pela primeira vez em maio de 2002 e a última publicação em dezembro de 2014. Seu cadastro conta com um total de 5.148 áreas. No Rio de Janeiro, o cadastro é elaborado pelo Instituto Estadual do Ambienta (INEA), tendo sua primeira edição em 2013 e última divulgação em 2014, com um total de 270 áreas cadastradas. O estudo sistematizou os resultados destes cadastros, conforme seu quantitativo e classificação, o que permitiu a análise posterior.

\subsection{Coleta de informações sobre as áreas contaminadas cadastradas em cada estado.}

Os dados e informações levantadas para a elaboração do panorama de áreas contaminadas da região sudeste, foram coletadas a partir do Cadastro de Áreas Contaminadas existentes nos Estados de São Paulo e Rio de Janeiro, o estado do Espirito Santo não possuía o cadastro até o momento da elaboração do presente artigo, este cadastro é uma importante ferramenta prevista na Resolução CONAMA 420, a qual estabelece diretrizes para o gerenciamento ambiental de áreas contaminadas, no sentido de dar publicidade sobre a identificação e principais características destas áreas. No estado de São Paulo, existe o Cadastro de áreas contaminadas e reabilitadas, elaborado pela Companhia Ambiental do Estado de São Paulo (CETESB), tendo sido divulgado pela primeira vez em maio de 2002 e última publicação em dezembro de 2014. No estado do Rio de Janeiro, existe o Cadastro de Áreas Contaminadas e Reabilitadas, emitido pelo Instituto Estadual do Ambienta (INEA), tendo sua primeira edição em 2013 e última divulgação em 2014. Por se tratar de um 
panorama que engloba três estados diferentes, houve a necessidade de alinhar as informações para uma mesma linguagem para que se pudessem comparar dados equivalentes entre os estados. Foram levantados e comparados dados de áreas contaminadas extraídos dos cadastros de cada estado em relação às atividades que ocasionaram a contaminação, a classificação das áreas, segundo sua situação relativa à etapa do gerenciamento de áreas contaminadas, comparativo com relação à evolução do numero de áreas contaminadas cadastradas e um comparativo dos principais contaminantes existentes nestas áreas.

\subsection{Comparativo da classificação das áreas segundo sua etapa do gerenciamento de áreas} contaminadas.

Para realizar o comparativo entres os estados da classificação/situação em que se encontram as áreas contaminadas de acordo com as diretrizes da CONAMA 420 e do gerenciamento de áreas contaminadas foram consultados os dados existentes nos cadastros de São Paulo e Rio de Janeiro. Para que se tornasse possível o comparativo de forma a avaliar dados em uma mesma linguagem, foi necessário realizar ajustes, pois no estado de São Paulo são considerados 06 tipos de classificações e no Estado de e Rio de Janeiro, são considerados 04 tipos de classificações, conforme Tabela 01 a seguir:

Tabela 01 - Classificação das áreas contaminadas nos estados de SP e RJ.

\begin{tabular}{|c|c|c|c|}
\hline Classificação Rio de Janeiro & Número de Áreas & Classificação São Paulo & Número de Áreas \\
\hline $\begin{array}{c}\text { Área Contaminada sob Intervenção - } \\
\text { ACI }\end{array}$ & 96 & $\begin{array}{c}\text { Área contaminada com risco confirmado } \\
\text { - ACRi }\end{array}$ & 631 \\
\hline $\begin{array}{l}\text { Área em Monitoramento para } \\
\text { Reabilitação - AMR }\end{array}$ & 35 & $\begin{array}{l}\text { Área contaminada em processo de } \\
\text { reutilização - ACRu }\end{array}$ & 87 \\
\hline $\begin{array}{c}\text { Área Contaminada sob Investigação - } \\
\text { AI }\end{array}$ & 130 & $\begin{array}{c}\text { Área contaminada sob investigação - } \\
\text { ACI }\end{array}$ & 1028 \\
\hline Reabilitadas - AR & 9 & $\begin{array}{l}\text { Área em processo de monitoramento } \\
\text { para encerramento - AME }\end{array}$ & 1204 \\
\hline- & - & $\begin{array}{c}\text { Área em processo de remediação - } \\
\text { ACRe }\end{array}$ & 1635 \\
\hline- & - & $\begin{array}{c}\text { Área reabilitada para uso declarado - } \\
\text { AR }\end{array}$ & 563 \\
\hline
\end{tabular}

Para um correto ajuste dos dados para uma comparação em uma mesma linguagem entres os estados, a classificação ACRi foi considerada equivalente a classificação ACI, as classificações 
ACRe, AME e ACRu formam consideradas equivalentes a classificação AMR e a classificação ACI equivalente a classificação AI, gerando a Tabela $\mathbf{0 2}$ a seguir.

Tabela 02 - Classificação das áreas contaminadas nos estados de SP e RJ com dados ajustados.

\begin{tabular}{|c|c|c|c|}
\hline Classificação Rio de Janeiro & Número de Áreas & Classificação São Paulo & Número de Áreas \\
\hline $\begin{array}{l}\text { Área Contaminada sob Intervenção - } \\
\qquad \mathbf{A C I}\end{array}$ & 96 & $\begin{array}{l}\text { Área Contaminada sob Intervenção - } \\
\qquad \mathbf{A C l}(\mathrm{ACRi})\end{array}$ & 631 \\
\hline $\begin{array}{l}\text { Área em Monitoramento para } \\
\text { Reabilitação - AMR }\end{array}$ & 35 & $\begin{array}{c}\text { Área em Monitoramento para } \\
\text { Reabilitação - AMR (ACRe, AME, ACRu) }\end{array}$ & 2926 \\
\hline Área Contaminada sob Investigação - Al & 130 & $\begin{array}{l}\text { Área Contaminada sob Investigação - Al } \\
\qquad(\mathrm{ACl})\end{array}$ & 1028 \\
\hline Reabilitadas - AR & 9 & Reabilitadas - AR (AR) & 563 \\
\hline
\end{tabular}

\section{RESULTADOS E DISCUSSÃO}

Resultados a partir da analise dos dados de classificação das áreas contaminadas entres os Estados de SP e RJ:

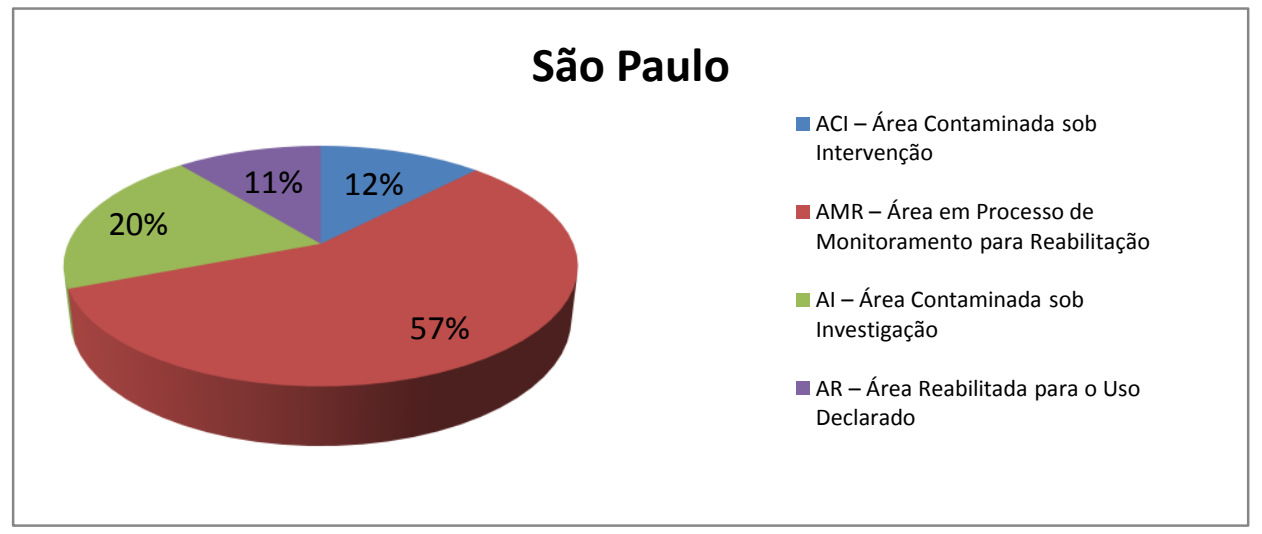

Gráfico 01 - Classificação das áreas contaminadas do Estado de São Paulo. 


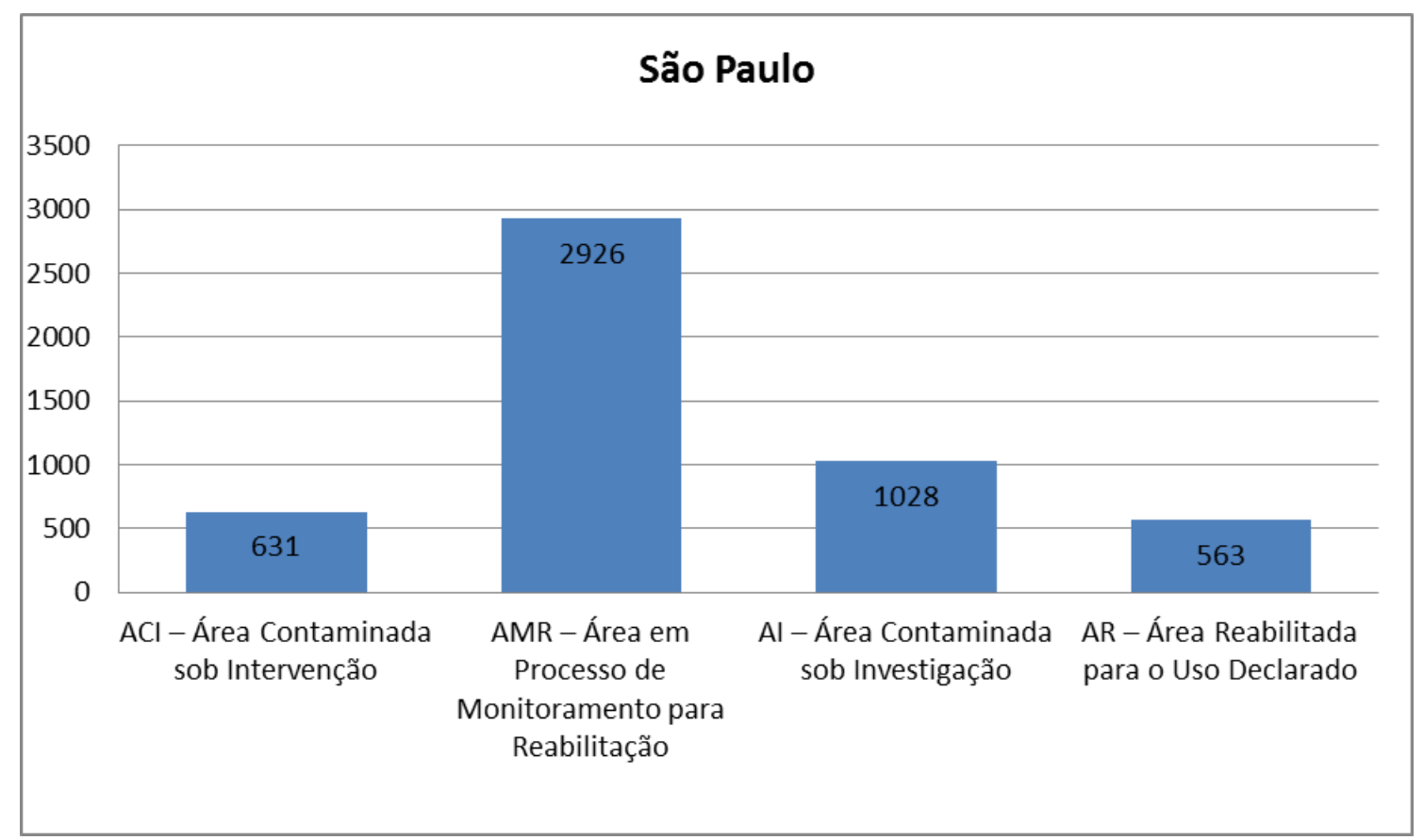

Gráfico 02 - Quantitativo de áreas contaminadas do Estado de São Paulo.

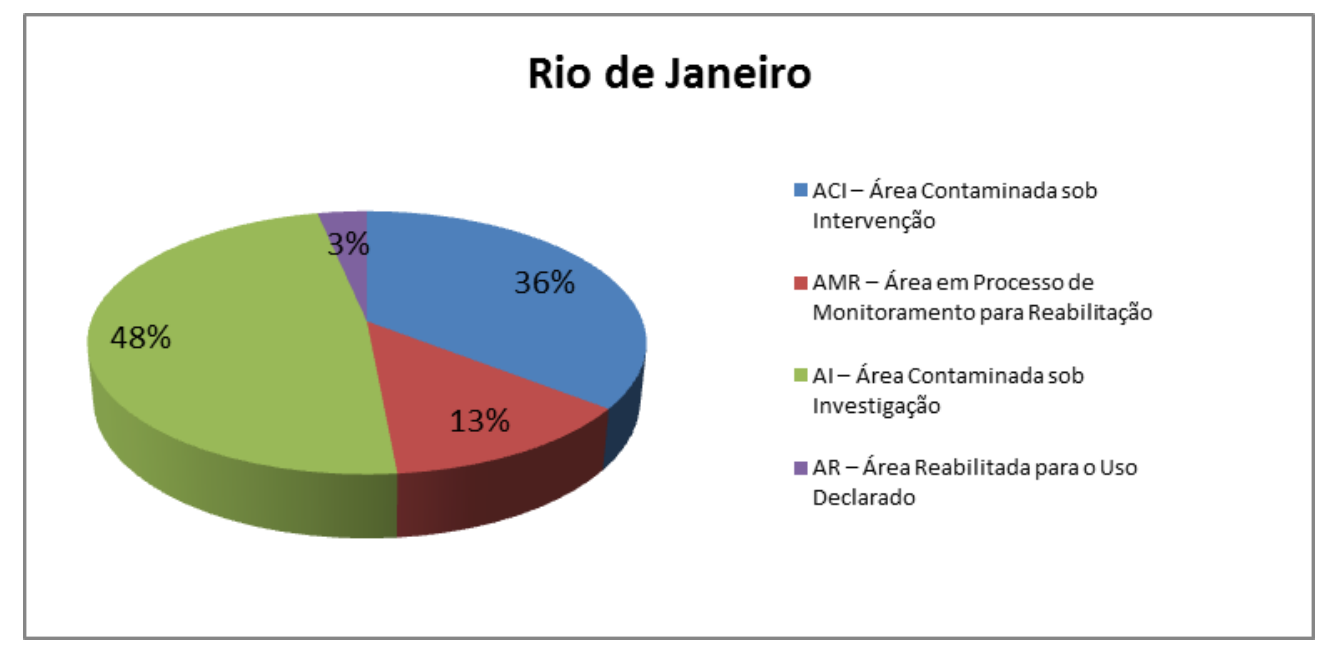

Gráfico 03 - Classificação das áreas contaminadas do Estado do Rio de Janeiro. 


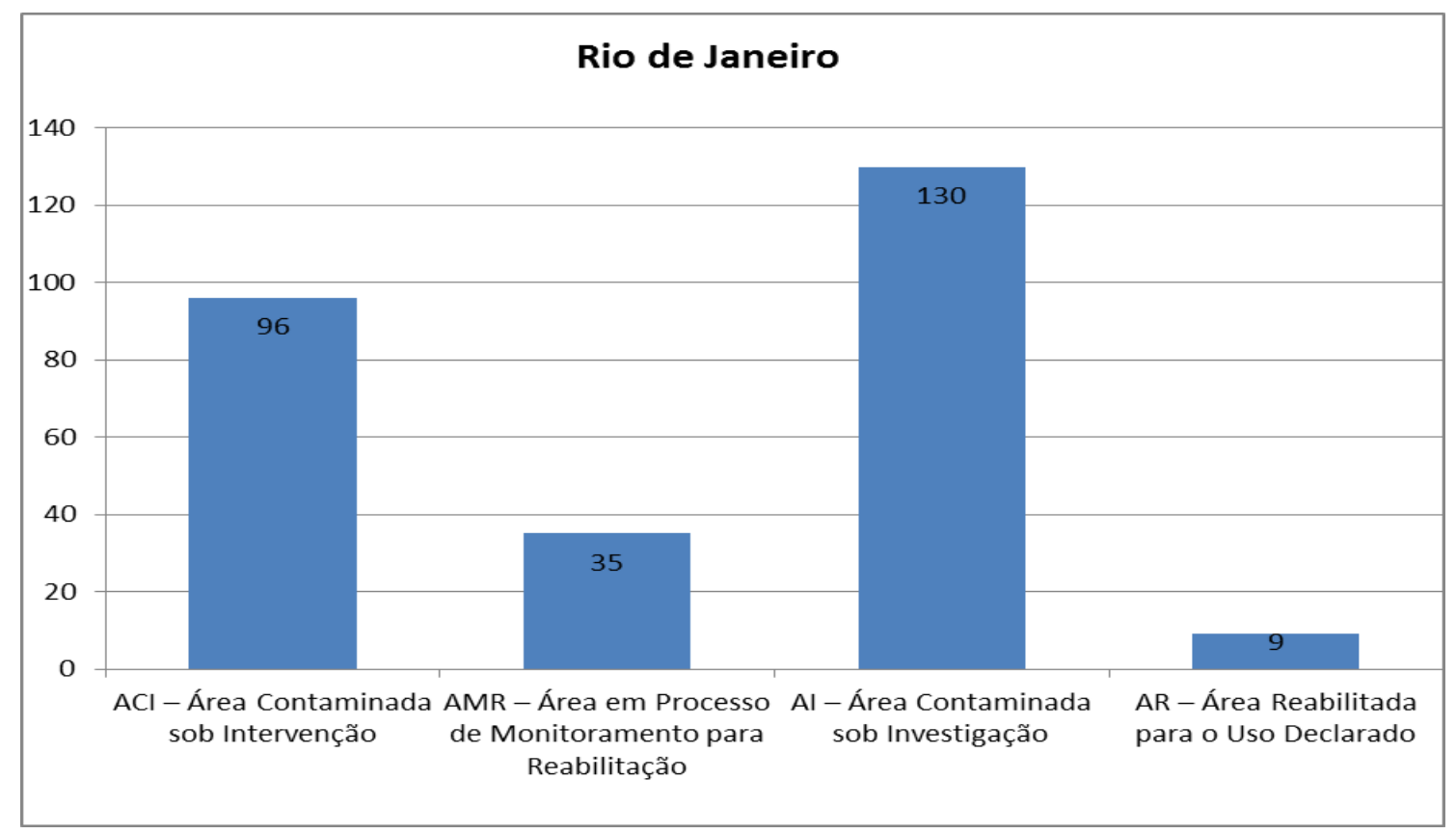

Gráfico 04 - Quantitativo de áreas contaminadas do Estado do Rio de Janeiro.

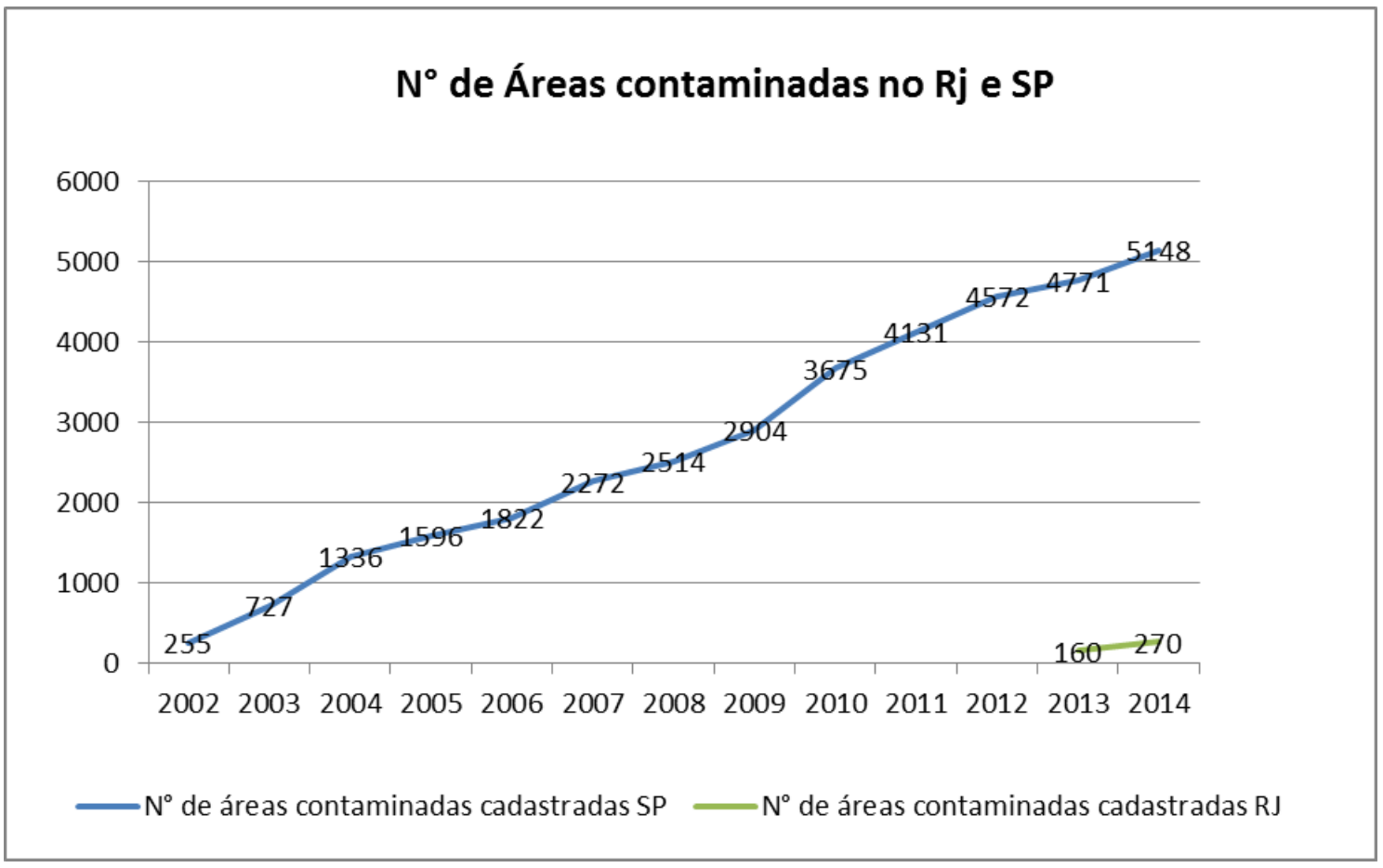

Gráfico 05 - Comparativo de áreas contaminadas entre os Estados do RJ e SP.

A análise dos gráficos acima demonstra que o pioneirismo de São Paulo resultou na maturidade na gestão de suas áreas contaminadas, traduzida no porcentual de AMR e AR, 68\% do total de registros. No Rio de Janeiro com atuação mais recente, os números mostram que o estado se estrutura para atingir os níveis do Estado de São Paulo, visto o elevado percentual - 48\% - de AI, 
etapa que precede as ações de remediação e que resultam no aumento de $\mathbf{A M R}$ e $\mathbf{A R}$. Estes três estados apontam o caminho e soluções para que os demais estados brasileiros conheçam e recuperem suas áreas contaminadas.

\section{CONCLUSÃO}

A partir deste comparativo fica claro a necessidade de ampliação dos estudos e aumento das exigências de levantamentos sobre a ocorrência de passivos ambientais no Estado do Rio de Janeiro, visto que ele é segundo maior PIB do Brasil, e com isso há a instalação de indústrias e atividades potencialmente poluidoras. Além disso, o mapeamento de áreas contaminadas é de suma importância para que a sociedade tenha ciência não apenas da áreas que estão em processo de reabilitação, mas também as reabilitadas para uso declarado, assim em casos de vendas de terrenos aonde tenham a ocorrência no passado de passivos ambientais possa entrar por exemplo na negociação e resolução sobre esse passivo.

Vale ressaltar que o Estado de São Paulo além de pioneiro tem sido um motivador e orientador para os demais Estados e o fato de tornar público o mapeamento realizado através de duas edições, até o momento, do Estado do Rio de Janeiro, reflete a necessidade de replicabilidade para os demais estados brasileiros. Apesar de não ser tema deste estudo, mas o Estado de Minas Gerais também já publicou o inicio do seu mapeamento de áreas contaminadas.

\section{REFERÊNCIAS}

CETESB (Org.). Relação de áreas contaminadas e reabilitadas no estado de São Paulo. São Paulo: Cetesb, 2014. 14 p.

INEA. Gerenciamento de Áreas Contaminadas do Estado do Rio Janeiro. Rio de Janeiro: Inea, 2014. $14 \mathrm{p}$.

CONAMA. Resolução $\mathbf{n}^{\mathbf{0} 420}$ - critérios e valores orientadores de qualidade do solo quanto à presença de substâncias químicas e estabelece diretrizes para o gerenciamento ambiental de áreas contaminadas por essas substâncias em decorrência de atividades antrópicas. CONAMA - Conselho Nacional de Meio Ambiente, 2009.

CONEMA Resolução $n^{\circ} 44$ - Dispõe sobre a obrigatoriedade da identificação de eventual contaminação ambiental no solo e na água subterrânea por agentes químicos, no processo de licenciamento ambiental. CONEMA - Conselho Estadual de Meio Ambiente, 2012. 
CETESB,GTZ.- Manual de Gerenciamento de áreas contaminadas. $2^{\mathrm{a}}$ ed. 2001. 389p. Projeto de Cooperação Técnica Brasil - Alemanha - CETESB - Companhia de Tecnologia de Saneamento Ambiental e GTZ, São Paulo, 2001.

Lei 13.577 - Dispõe sobre diretrizes e procedimentos para a proteção da qualidade do solo e gerenciamento de áreas contaminadas. Estado de São Paulo, 2009. 